\title{
KawaWiki: A Semantic Wiki Based on RDF Templates
}

\author{
Kensaku Kawamoto, Yasuhiko Kitamura, and Yuri Tijerino \\ Kwansei Gakuin University \\ 2-1 Gakuen, Sanda-shi, Hyogo 669-1337, JAPAN \\ \{kkensaku, ykitamura\}@ksc.kwansei.ac.jp, yuri@tijerino.net
}

\begin{abstract}
A Semantic Wiki is a collaborative Semantic Web authoring system based on the Wiki framework. It provides a scheme where anonymous users on the Internet can collaborate with each other to build a Semantic Web site. Contrasting to traditional Wikis, it is not easy for end users to author Semantic Wiki pages from scratch without knowledge of the complex RDF/OWL syntax and of ontologies to share semantic information. We propose a new Semantic Wiki system called "KawaWiki" on which end and expert users can collaborate to build a Semantic Web site. KawaWiki generates RDF and Wiki pages based on its RDF templates and validates their consistency with the RDFS description. The RDFS description can be created by importing Web ontologies on the Internet. KawaWiki aims at providing a scheme where end users, expert users and ontologists seamlessly collaborate to publish semantically consistent RDF and Wiki pages in a Wiki way.
\end{abstract}

\section{Introduction}

The Semantic Web was proposed by Tim Berners-Lee at $\mathrm{W} 3 \mathrm{C}$ as a standard for next generation Web systems [1]. It's aim is to enable computers to retrieve and gather information automatically from the Web by adding semantic annotations to Web pages.

Currently, the Semantic Web enjoys much attention with significant efforts taking place in academia and industry. At present, most of Semantic Web information is provided by academic institutes or commercial companies as an advanced form of Web services, but we need tools with which even an end user, who is not an expert on Semantic Web technology, can easily disseminate Semantic Web information. We believe such tools could enhance the proliferation and usability of the Semantic Web across the Web user community.

WikiWikiWeb (or Wiki, in short) is a tool developed by Ward Cunningham in 1990's for authoring Web pages col- laboratively [2]. Using a Wiki system, it is possible to develop a Web content collaboratively by creating and updating Wiki pages directly through Web browsers. Currently a number of Wiki platforms have been developed and are available to the general Web community. Most Wiki systems provide a simple mark up language to enable content creation in Wiki pages. Wikipedia.org, which uses the Mediawiki platform [3] is the most well-known Web site developed by using the Wiki framework.

Recently efforts to integrate Semantic Web and Wiki technologies have become popular $[4,5,6]$ and a new research field called Semantic Wiki has been born. To this end, several frameworks have been proposed, which can be categorized as follows.

1. Automated RDF meta data generation. RDF data is automatically generated for meta data provided by users for Wiki pages. Many conventional Wiki systems such as PukiWiki ${ }^{1}$ or YukiWiki ${ }^{2}$ already include this function ality. Although users do not need to know about the RDF syntax, these systems provide automated RDF data generation, for user defined meta data. The RDF descriptions provided by these systems are limited to meta data for Wiki pages such as the title, the update date and time, the links, and so on.

2. Automated RDF generation from intermediate representations. RDF data is automatically generated through special tags and commands inserted by users throughout the content. Peri Peri ${ }^{3}$ and Rhizome Wiki ${ }^{4}$ are examples of this category. The users have to insert special semantic tags or equivalent components by themselves. This requires users to learn the constructors in advance, which may require a step learning curve.

3. User-generated RDF annotations. RDF data is di-

\footnotetext{
${ }^{1}$ http: //pukiwiki.org/

${ }^{2}$ http: //www. hyuki.com/yukiwiki/

${ }^{3}$ http://www.srcf.ucam.org/ cjp39/Peri/

${ }^{4}$ http: //rhizome.liminal zone.org
} 
rectly provided by users in raw form. Platypus $\mathrm{Wiki}^{5}$ is an example of this category. The users have to insert raw RDF/XML data by themselves, so the system may be viewed as a RDF editor on a Web browser. Of course, this method depends on users becoming familiar with the RDF syntax, which requires a much steeper learning curve, making it very difficult for general end user to provide such annotations.

All of these categories require some degree of expertise on the Semantic Web from part of the users. Although the automated RDF generation approaches do not require extensive Semantic Web expertise, it still requires knowledge about ontologies or at least about how to reuse conceptual descriptions provided by others and how to publish new ones for others to reuse. On one hand, if the generated RDF descriptions are based on stand-alone concepts, the descriptions would serve no utility for the Semantic Web and would be nothing more than simple meta data. On the other hand, if the user provides descriptions or annotations by reusing concepts described by others or found in generally used ontologies, the descriptions become integral part of the Semantic Web. In this paper, we propose a new Semantic Wiki system called "KawaWiki", which takes the later approach to enable end-users to provide Semantic Web descriptions and annotations on Wiki content. This system utilizes RDF templates to prevent users from having to learn the complicated RDF syntax, and to allow even end users to generate RDF data through simple forms on a browser. KawaWiki also provides a validation checking mechanism to guarantee the syntactic and semantic integrity of Wiki/RDF pages based on the templates and RDFS, which in addition enable reuse of conceptual descriptions found in the Semantic Web.

\section{KawaWiki}

Wiki users can be classified into two groups: expert users who can create Wiki pages from scratch and end users who can only rewrite and update the Wiki pages that have been already created by others. Not all the users can create Wiki pages from scratch. Rather, some users create Wiki pages, and others just update them. We can view the former as expert users and the latter as end users.

KawaWiki explicitly exploits collaborations between these two groups; end users who know nothing or little about Semantic Web descriptions and expert users who have basic knowledge about it. This follows the end user paradigm of current Wiki systems, in which end users can be classified into these two groups.

In KawaWiki, we further classify expert users into expert users with knowledge about the syntax of RDF/OWL, and

\footnotetext{
${ }^{5}$ http://platypuswiki. sourceforge.net/
}

ontologists with knowledge about ontologies, which enable them to reuse and share semantic information widely and effectively on the Semantic Web. KawaWiki aims at providing a scheme where these three groups; end users, expert RDF/OWL users and ontologists, collaborate to create and publish Semantic Web information in a Wiki way as shown in Figure 1.

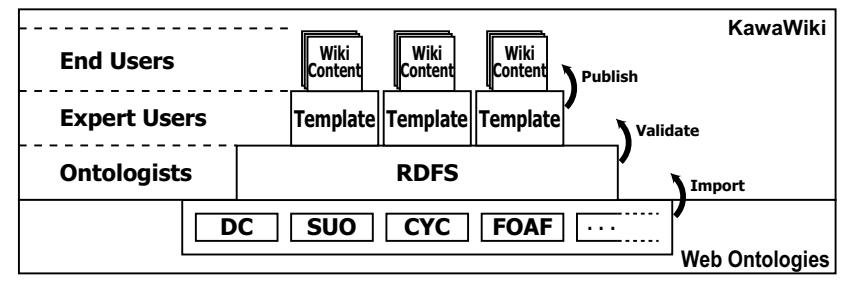

Figure 1. KawaWiki End User Architecture.

\subsection{Authoring RDF and Wiki pages using RDF templates}

In essence, Semantic Web consists of a Web of interwoven semantic tags that are processable and understandable by computers. RDF is a language that is widely used to describe semantic information through a triples consisting of a subject, a predicate and an object. This allows semantic information to be represented as a set of interconnected triples.

RDF is generally defined in the XML language, so it may be referred to as RDF/XML. KawaWiki uses the RDF/XML syntax as its Semantic Web description language. However, it is not easy for end users to author the RDF pages without knowledge of the complex RDF syntax. To reduce the burden of end users, KawaWiki introduces RDF templates to conceal the RDF syntax from the end users.

As shown in Figure 2, we use a RDF template to publish a RDF page. RDF tags have been prepared in the templates and the instance parts are left to be filled. KawaWiki enables expert RDF users and ontologists to generate and edit Web forms based on the RDF template, which end users then complete by inserting instance data into the form entry components. Finally KawaWiki generates the RDF description page based on the RDF template and the inserted data. It also generates a Wiki page at the same time.

We show an example of a template in Fig. 2. This template is used to generate RDF/Wiki pages about a person involved in a particular project using the FOAF standard to illustrate how templates can make references to external resources, including ontologies.

KawaWiki templates are written in RDF and consist of three parts. The first part (lines 2-9) defines the name space of the template, just as in usual RDF documents. The sec- 


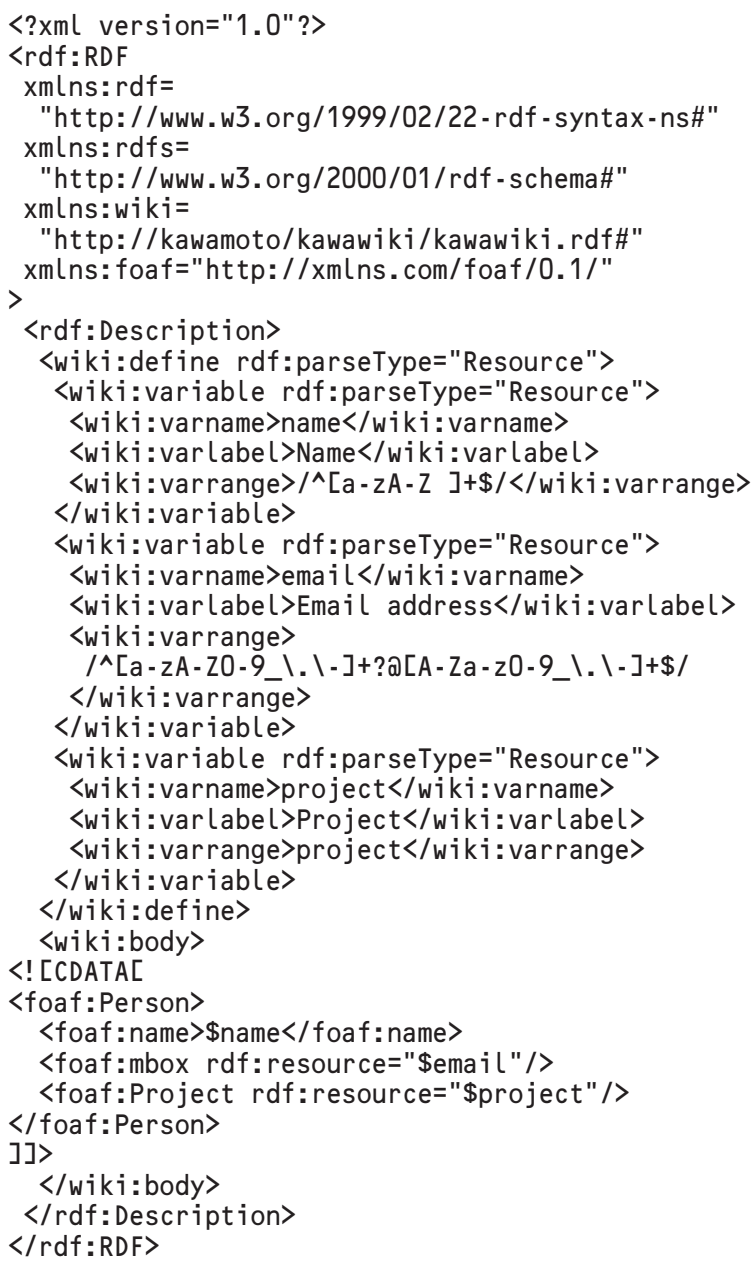

Figure 2. Example of RDF template.

ond part (lines 12-30) defines the declaration of variables. A literal or a resource is set as a variable in KawaWiki. This template uses three variables name for the name of person, email for the Email address of person, and project for the project in which the person is involved. The definition of a variable consists of the name, the label, and the range. The label is a description of the variable. If the variable takes a literal, the range specifies its value range in the Regular Expression. For example, line 16 specifies variable name takes alphanumerics of any length. If the variable takes a resource, its value range specifies the name of resource. Line 28 specifies variable project takes a resource, which is a project Wiki page defined a priori in a KawaWiki ontology.

The third part (lines 31-39) defines the body of the template. It is the source for RDF data instantiations. From this description, a form with blanks is generated for users to fill in. In this example, blanks for \$name, \$email and $\$$ project generate the appropriate form components, which the users needs to fill in.

To create a Wiki page using this template, a form appears on a Web browser as shown in Fig. 3(a) for the user to fill in. Property Name and Email address are left blank on this form, so the user inserts data in the textbox and the data instantiates the variables name and email. The property project refers to a project resource and can be set by selecting an item from a collection of project candidates from a pull-down menu. In this example, the collection of projects has been previously defined by the ontologist in a conceptual hierarchy and illustrates how the KawaWiki framework not only allows reference to external ontologies, but also to internally defined ones. When the user pushes the "Write" button, a Wiki page appears as shown in Fig. 3(b). If the user clicks on the RDF icon on the bottom-right of the Wiki page, the generated RDF data of the Wiki page appears as shown in Fig. 3(c).

Although, the KawaWiki framework allows expert users and ontologies to create RDF and ontologies through a Web browser in a Wiki way, this functionality has not been implemented, yet.

\subsection{Validating RDF templates using RDFS}

A Wiki can be thought of as a collaborative authoring tool that allows easy generation of Web pages. With this easy-to-use tool, users can create and update Web pages at any time from any place through the Internet. They can write whatever they like on the pages and there is basically no scheme to guarantee their semantic integrity. On the other hand, the Semantic Web is based on a rigid formal foundation and can be used to guarantee the integrity and consistency of Web content. Therefore, the semantic integrity and consistency of Semantic Wikis is an interesting research topic.

KawaWiki uses a RDFS description to validate RDF templates as illustrated in Fig. 4. Whenever we create or update a RDF template, KawaWiki checks its semantic consistency with the RDFS description. If it finds any inconsistency with the RDFS, it indicates an error and suspends the creation or the updates.

KawaWiki also validates the syntactic integrity of RDF/Wiki pages through the use of templates. Validations are generated automatically from templates and data inserted by users. The input data is validated by referring to the variable range description in the template as discussed in section 2.1.

If KawaWiki finds any semantic/syntactic errors in templates or RDF/Wiki pages, it shows a warning on the browser and suspends the update. 


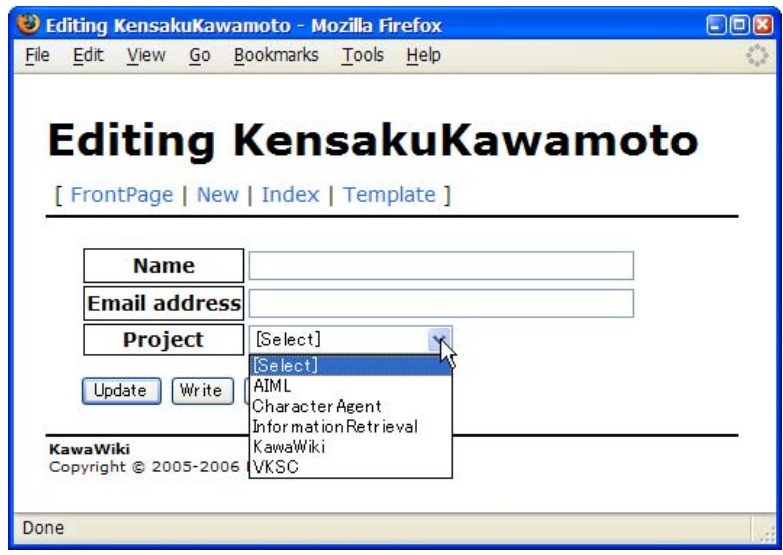

(a) Form generated from a template.

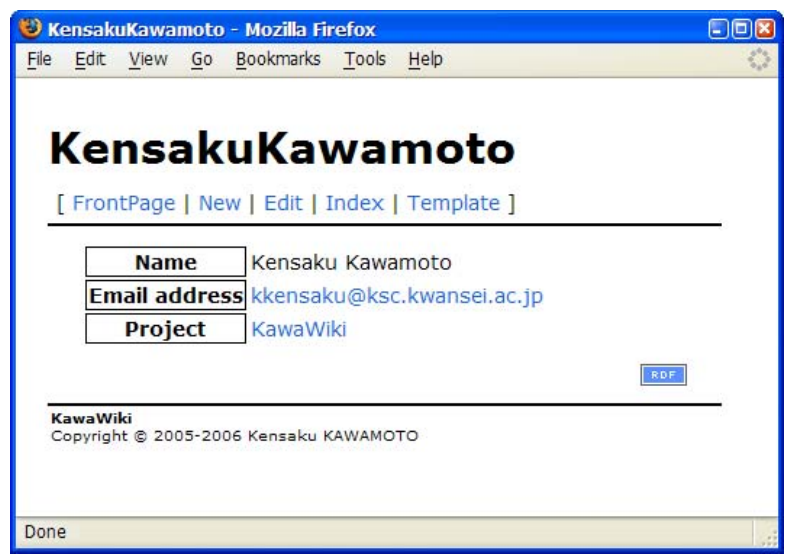

(b) Wiki page generated from a template and inserted data.

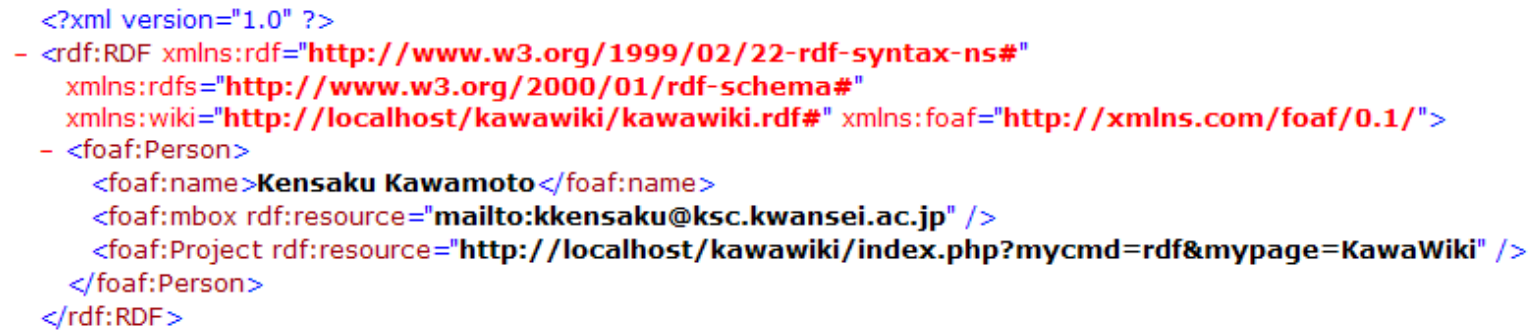

(c) RDF data generated from a template and inserted data.

Figure 3. Snapshots of KawaWiki.

\subsection{References to Web ontologies}

It is important that the vocabulary used in RDF pages is consistent with common ontologies such as DC, SUO, CYC, FOAF, and so on, so that the RDF pages and descriptions can be shared with other users and Semantic Web systems on the Internet. KawaWiki provides functionality to make reference to these ontologies in the RDFS ontologies. As mentioned previously, RDF pages are consistent with the RDFS through the RDF templates. Consequently, KawaWiki content is consistent with the common, concepts, relations and constrains found in common ontologies in the Semantic Web.

If the available ontologies do not provide the semantics needed to describe a particular content in a RDF Wiki page, KawaWiki allows the ontologist to create new ones and incorporate them in the RDFS/OWL ontology.

\subsection{Implementation}

A prototype of KawaWiki has been developed and is currently being improved. It is written in PHP and is based on YukiWikiMini [7], which was originally written in Perl. It employs RAP (RDF API for PHP) for handling RDQL queries and RDF. We are developing a Web ontology integration mechanism at present.

\section{Conclusion and Future Work}

KawaWiki aims at providing a scheme where end users, expert users, and ontologists collaborate to publish Semantic Web information in a Wiki way. We have developed a RDF template system to conceal the complex RDF syntax from end users and a validation checking mechanism to guarantee the consistency of RDF data.

In our future work, we will enhance KawaWiki to allow the three types of users collaborate more elaborately. An interesting research topic of Semantic Wiki is how a number of users can build a semantically consistent Semantic Web site. If KawaWiki can point out inconsistent parts of the site automatically to the users, they may be able to fix them efficiently. At present, validation checking concerns only the consistency between RDF templates and the RDFS description, so we need to enhance the checking scheme by introducing inference mechanism as well. 


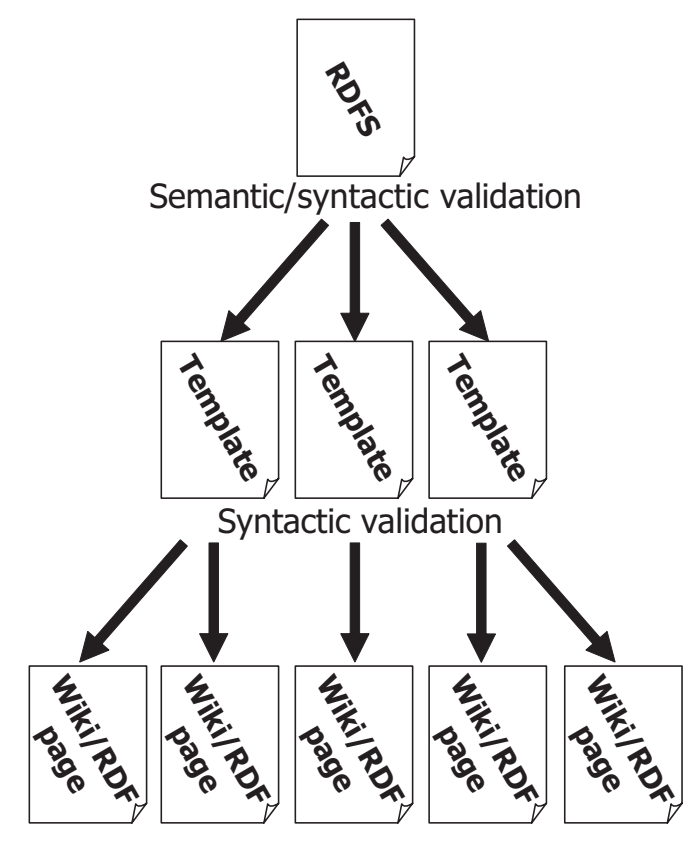

Figure 4. Validation checking in KawaWiki.

\section{Acknowledgement}

This work is partly supported by the Grant-in-Aide for Scientific Research (No.17300050 and No.16200010) from Japan Society for the Promotion of Science.

\section{References}

[1] Tim Berners-Lee, James Hendler, and Ora Lassila. The Semantic Web: A new form of Web content that is meaningful to computers will unleash a revolution of new possibilities. Scientific American, May 17, 2001.

[2] Bo Leuf and Ward Cunningham. The Wiki Way: Collaboration and Sharing on the Internet, Addison-Wesley Professional, 2001.

[3] Wikipedia contributors, Mediawiki:cite text, Wikipedia, The Free Encyclopedia, 2006. [Online; accessed 22September-2006].

[4] Roberto Tazzoli, Paolo Castagna, and Stefano Emilio Campanini. Towards a Semantic WikiWikiWeb. Poster Track, 3rd International Semantic Web Conference pp. 7-11, November 2004.

[5] Hideaki Takeda and Muljadi Hendry. Towards Semantic MediaWiki (in Japanese), SIG-SWO-A404-06, 2004.
[6] David Aumueller. Semantic authoring and retrieval within a Wiki. 2nd European Semantic Web Conference, 2005.

[7] Hiroshi Yuki. Wiki Nyumon (in Japanese), Impress, 2004. 